\title{
The Destruction of a Compact Group of Galaxies
}

\author{
Rachel A. Pildis \\ Department of Astronomy, University of Michigan, Ann Arbor MI 48109-1090; \\ pildis@astro.lsa.umich.edu
}

\begin{abstract}
The very high apparent galaxy densities in Hickson compact groups (HCGs) should produce copious amounts of galaxy-galaxy interaction, if these groups are bound dynamical units. I examine the evidence for such interactions in HCG 94, a group of seven galaxies with an envelope of diffuse optical light and an extremely high X-ray luminosity. Using ROSAT HRI data and deep three-color optical imaging of the group, I find that both the hot $(k \mathrm{~T} \sim 4 \mathrm{keV})$ gas and the diffuse optical light trace the same elongated potential well, which is offset from the galaxy distribution. The luminosity and colors of the diffuse optical light imply that the group galaxies are being ripped apart to form a cD-type remnant galaxy. The similarity in shape of the X-ray and optical contours may mean that this destruction process may require several billion years.
\end{abstract}

Subject headings: galaxies: clusters: individual-galaxies: interactions

\section{Introduction}

Compact groups of galaxies provide a puzzle: galaxies that are in such close proximity should merge together rapidly, yet there are many compact groups and few obvious group remnants such as isolated bright ellipticals. Some have questioned whether compact groups are real, physical groupings, but studies of samples such as the Hickson compact groups (HCGs; Hickson 1982) have shown that most group galaxies have accordant redshifts (Hickson 1993) and many of them have morphological signs of interaction (Mendes de Oliveira \& Hickson 1994). In addition, recent X-ray investigations (Ebeling et al. 1994, hereafter EVB; Pildis et al. 1995a, hereafter Paper I) demonstrate that a significant fraction of elliptical-rich HCGs have extended emission consistent with hot gas in a group potential (7 of the 8 groups in Paper I with spiral fractions of under $50 \%$ have extended emission), indicating that they are likely to be gravitationally bound. 
In this Letter, I present new evidence that one X-ray-bright Hickson compact group- $-\mathrm{HCG} 94$ - may be near the end of its life as a group, destroying itself to form a remnant galaxy that may resemble a cD. HCG 94 is a group with seven members, all but one of them being either elliptical or S0 (Fig. 1-throughout this paper, I use the galaxy designations of Hickson $[1982,1993]$ and assume $\mathrm{H}_{0}=50 \mathrm{~km} \mathrm{~s}^{-1} \mathrm{Mpc}^{-1}$ ). The diffuse optical light surrounding the two brightest galaxies has been known for some time (Hickson 1993), but its extent has not been mapped. This diffuse light makes HCG 94 one of the Hickson compact groups most likely to be close to a merger of its member galaxies.

Serendipitously, this group appeared in a ROSAT PSPC observation of another group, HCG 93. No spatial analysis was possible since HCG 94 was $30^{\prime}$ off-axis, but spectral analysis gave a temperature of $k \mathrm{~T}=3.7 \pm 0.6 \mathrm{keV}$ and a luminosity of $\mathrm{L}(0.07-3.0 \mathrm{keV})$ $=7.19 \times 10^{43} \mathrm{erg} \mathrm{s}^{-1}$ (Paper I-where we assumed a Raymond-Smith thermal model with absorption due only to the Galactic neutral hydrogen in the direction of this group and an abundance for the hot gas of $50 \%$ solar.). This is hotter and brighter than other X-ray-detected Hickson groups, which have temperatures of $\sim 1 \mathrm{keV}$ and luminosities of $10^{41-42} \operatorname{erg~s}^{-1}$ (EVB, Paper I).

The observations to be presented here are a new ROSAT High Resolution Imager (HRI) observation, important because the galaxies in this group are too close together to be resolved by the PSPC, and deep three-color $(B V R)$ imaging, obtained as part of a larger survey for low-surface-brightness optical features in HCGs (Pildis et al. 1995b, hereafter Paper II). Both sets of observations reveal that the group potential is offset from the distribution of galaxies in HCG 94, and that the destruction of the group is proceeding slowly enough for the stars in the diffuse light to follow the same potential as the hot gas.

\section{Observations}

The optical observations were obtained in August 1994 with the 2.4m Hiltner telescope at the Michigan-Dartmouth-M.I.T. Observatory on Kitt Peak, Arizona. HCG 94 was imaged in $B, V$, and $R$ using a $1024^{2}$ pixel back-illuminated Tektronix CCD with a plate scale of $0.275^{\prime \prime}$ pixel $^{-1}$. Calibrations were made using observations of standard stars from Landolt (1992), and all magnitudes and colors have been corrected for Galactic extinction. Details of the reduction procedure for this and other optical observations of a sample of HCGs will be given in Paper II. For HCG 94, the errors in the magnitudes are less than $0.05 \mathrm{mag}$ and the errors in the colors are on the order of $0.05 \mathrm{mag}$. 
The X-ray observations of HCG 94 by the ROSAT HRI were taken in December 1994, with a total live time of 30,045 seconds. The data were corrected for vignetting using the PROS package in IRAF. No signal was found in PHA channels 1 and 10-16 (as is often found with HRI observations), and thus those channels were excluded from the analysis. In order to examine the spectral hardness of the emission from HCG 94, I created two images: one using PHA channels 2-4, which is optimized for temperatures of less than $1 \mathrm{keV}$, and one using channels 5-9, for higher temperatures.

\section{Results}

Comparison of the two HRI maps reveals strikingly different structures for the high and low temperature X-ray-emitting gas in this group. Chi-squared fits to the low temperature data show that this fainter emission is circularly symmetric $(Q=0.81)$ about galaxy a. A similar fitting procedure applied to the high temperature map demonstrates that this bright emission is best fit by an ellipse of $\epsilon=0.35(Q=0.79)$ with the major axis pointing north-south, centered $25^{\prime \prime}$ south of galaxy a and almost directly east of galaxy b. The centers are determined to within $4^{\prime \prime}$, so the displacement between the centers of the two components is significant.

After determining the background level by examining the radial profile of the emission (the HRI produces very flat images, making this determination unproblematic), the background was subtracted. Using a simplex program, I fit the radial surface brightness profiles of both maps with the standard hydrostatic-isothermal beta model (Cavaliere \& Fusco-Femiano 1976):

$$
S(r)=S_{0}\left(1+\left(\frac{r}{r_{\text {core }}}\right)^{2}\right)^{-3 \beta+0.5}
$$

convolved with the point-spread function of the HRI. The background-subtracted radial surface brightness profiles are shown in Fig. 2, and the fitting results are listed in Table 1. This spatial analysis is very similar to that carried out on PSPC observations of HCGs, as described in Paper I. While I used elliptical isophotes to fit a beta model to the high temperature component, using circular isophotes increases $\chi^{2}$ but does not change the fitted parameters by more than $1 \sigma$.

The HRI has a very limited capability to provide spectral information. From the relative count rates in the two images (see Table 1) and the HRI's low sensitivity to high energy X-rays $(k \mathrm{~T} \gtrsim 1 \mathrm{keV})$, one can determine that the high temperature component 
dominates, which is consistent with the $3.7 \mathrm{keV}$ temperature measured with the PSPC. Since the low temperature component is centered on galaxy a and has a value of $\beta$ consistent with the values found for elliptical galaxies, it is likely to also have a temperature typical for hot gas in ellipticals: $0.5-1.0 \mathrm{keV}$. The presence of this relatively cool component means that the PSPC temperature of $3.7 \mathrm{keV}$ is probably an underestimate of the temperature of the hot intragroup medium (whose $\beta$ value is similar to that of galaxy clusters). Thus, as a conservative estimate, I adopt $k \mathrm{~T}=4 \mathrm{keV}$ for the intragroup gas for the remainder of this Letter.

The diffuse optical emission appears to have the same shape and center as the high temperature X-ray emission, though its extent is smaller (Fig. 3). The galaxies in this group are generally to the north and east of the diffuse light and the hard X-ray emission (compare with Fig. 1). To a surface brightness of $26.5 \mathrm{~V}$ magnitudes $\operatorname{arcsec}^{-2}$, the north-south extent of the light is $2.4^{\prime}(0.17 \mathrm{Mpc})$ and its ellipticity is $\epsilon=0.3$ (similar to that of the X-ray emission). Its surface brightness profile is fairly flat, with the brightest emission located southeast of galaxy b $\left(22.7 \mathrm{~V}\right.$ magnitudes $\left.\operatorname{arcsec}^{-2}\right)$ and fainter emission found north and west of galaxy a $\left(23.6 \mathrm{~V}\right.$ magnitudes $\left.\operatorname{arcsec}^{-2}\right)$. The outer edges of the envelope are somewhat bluer than its central regions, but the color ranges are small: $B-V=0.95-1.10$ and $V-R=0.55-0.65$. Its $B-V$ colors are slightly redder than a typical elliptical galaxy $(B-V=0.8-1.0)$, but its $V-R$ colors are in the elliptical range of $0.5-0.7$ (Gregg 1989). Galaxies a and b both have blue gradients with increasing radius, from central colors of $B-V=1.20$ and $V-R=0.75$ to colors equal to those of the inner envelope. The red $B-V$ colors may indicate the presence of dust in this system.

In order to discriminate between overlapping galaxy light and diffuse light in the group potential, I ran an ellipse-fitting program on the cores of the brightest two galaxies, and then forced $r^{1 / 4}$ profiles with the same ellipse parameters for their envelopes (details in Paper II). The two model galaxies thus produced were then subtracted from the images in all three filters. I find that the total luminosity of the diffuse envelope is $-23.7 \mathrm{in} \mathrm{V}$, or $2.4 \times 10^{11} \mathrm{~L}_{\odot}$. If the two galaxies are included $\left(\mathrm{M}_{\mathrm{V}}=-22.9\right.$ and -22.3 , respectively), the system luminosity is -24.3 in $\mathrm{V}, 4.3 \times 10^{11} \mathrm{~L} \odot$.

\section{Implications}

If the galaxies in HCG 94 are merging to form an isolated cD-type elliptical, then the properties of the diffuse X-ray and optical emission should be comparable to those of $c \mathrm{D}$ envelopes. Schombert (1988) found that $\mathrm{cD}$ envelopes in his large sample have colors 
of $B-V=1.1-1.3$ and small to non-existent color gradients, similar to what is seen in HCG 94. He also found a strong correlation between cluster X-ray luminosity and optical envelope luminosity. Using that relation, the X-ray luminosity of HCG $94\left(7.2 \times 10^{43} \mathrm{erg}\right.$ $\left.\mathrm{s}^{-1}\right)$ corresponds to a $\mathrm{cD}$ envelope with $\log \left(\mathrm{L}_{\mathrm{L}} \mathrm{L}_{\odot}\right)=11.1-11.5$. The measured value for HCG $94, \log \left(\mathrm{L} / \mathrm{L}_{\odot}\right)=11.4$, is in that range.

Another way to compare the diffuse light in $\mathrm{HCG} 94$ to that in $\mathrm{cD}$ envelopes is to examine the difference between the the total magnitude of the system and the magnitude of the galaxy if it did not have an envelope. Using the models mentioned above for galaxies $\mathrm{a}$ and $\mathrm{b}$, this difference is -0.95 if $\mathrm{a}$ and $\mathrm{b}$ are together considered to be the central galaxy, and -1.27 if only galaxy a is used. Schombert (1986) made histograms of this difference for both brightest cluster members (BCMs) and $\mathrm{cDs}$, and found values ranging from 0 to -1.7 , with average values of roughly -0.6 for BCMs and -1.0 for cDs. Thus, HCG 94 appears to have an average to bright envelope as compared to central galaxies of clusters.

HCG 94 is not the sole known example of a system caught in the act of destroying itself. Schneider and Gunn (1982) found a similar system that they considered to be a nascent $\mathrm{cD}$ galaxy. This system, $\mathrm{V} \mathrm{Zw} \mathrm{311,} \mathrm{is} \mathrm{a} \mathrm{diffuse} \mathrm{halo} \mathrm{of} \mathrm{optical} \mathrm{light} \mathrm{surrounding}$ 9 early-type galaxies in the center of the poor cluster Abell 407. It has a color of $(g-r)$ $=0.44$ (equivalent to a typical elliptical galaxy color of $B-V=0.95$ [see Schneider et al. 1983]) and an absolute magnitude of -23.3. As in HCG 94, the brightest galaxy in V $\mathrm{Zw} 311$ is a strong radio source and the X-ray luminosity of Abell $407-5.8 \times 10^{43} \mathrm{ergs} \mathrm{s}^{-1}$ (Burns et al. 1994) - is almost identical to that of this Hickson group. V Zw 311 differs from the central part of HCG 94 in that there are more galaxies within its envelope, the system containing it has many more galaxies than an HCG (its radial velocity dispersion is $590 \mathrm{~km}$ $\mathrm{s}^{-1}$, while that of HCG 94 is $480 \mathrm{~km} \mathrm{~s}^{-1}$ [Hickson 1993]), and it may be at a more advanced stage of its evolution since its envelope is symmetric about the galaxy distribution. Thus, V Zw 311 may give us an idea of what HCG 94 may look like in the future as its destruction proceeds and galaxies continue to fall into it.

The "fossil group" discovered by Ponman et al. (1994) also has some intriguing similarities with HCG 94. This apparently isolated elliptical galaxy has an absolute magnitude of $\mathrm{M}_{\mathrm{V}}=-23.5$ and an X-ray luminosity of $4.5 \times 10^{43} \mathrm{erg} \mathrm{s}^{-1}$, as well as a rather red $V-R$ color of 0.8 . The outer envelope of the galaxy is even redder, which differs from the behavior of both ordinary ellipticals and HCG 94. The fossil group may provide a better comparison to HCG 94 than V Zw 311 since it is not a member of even a poor cluster, but appears to be isolated.

Some have speculated that HCG 94 is not actually an isolated group, but the core of a poor cluster that is interacting with Abell 2572, a cluster $16^{\prime}(1.2 \mathrm{Mpc})$ east at a 
similar redshift (EVB). However, isolation was one of the criteria that Hickson (1982) used to choose his groups, and the hot gas in HCG 94 is elongated in a north-south direction, showing no signs that a deep potential to the east is affecting it. This group may have an anomalously hot and bright intragroup medium, but there is no a priori reason to define it as a cluster core rather than simply a group with extreme X-ray properties.

Determining what the timescale of the destruction of HCG 94 is could answer some of the continuing questions about the lifetime of a compact group. The median crossing time of HCGs is only $0.016 \mathrm{H}_{0}^{-1}$ (Hickson et al. 1992), but simulations, especially those including a common dark matter halo, demonstrate that compact groups can survive up to $10^{9}$ years (e.g., Mamon 1987; Barnes 1989; Bode et al. 1993), or even longer if a spectrum of galaxy masses is used (e.g., Governato et al. 1991). Even the most optimistic theories still give merger times that are considerably shorter than a Hubble time, however. Such short lifetimes imply that compact group remnants, which are predicted to be "dynamically ordinary" - albeit isolated - ellipticals, should be common (Barnes 1989). Barnes (1989) attempted to explain the lack of such remnants by assuming that they are swept quickly into richer enviroments by the growth of gravitational clustering, but this would require the somewhat artificial assumption that the timescales of intragroup and intercluster merging are equal.

In HCG 94, the stars that make up the diffuse light appear to fill the same potential as the dissipative, X-ray-emitting gas does, implying that the potential well is not changing on a very rapid time scale. The crossing time for stars in the optical envelope is roughly $3 \times 10^{8}$ years (for velocities on the order of the radial velocity dispersion of HCG 94); to smooth out the distribution of stars would require a few crossing times, or over $10^{9}$ years. Since the sound-crossing time for $4 \mathrm{keV}$ gas in the same volume is only $1.6 \times 10^{8}$ years, the group potential must have been relatively constant for at least $10^{9}$ years. The free-free cooling time for the hot gas, which has an average electron density of $1.3 \times 10^{-2} \mathrm{~cm}^{-3}$, is $1.6 \times 10^{9}$ years, so the assumption that hot gas has filled this potential for $\sim 10^{9}$ years is not unreasonable. The long timescales seen for HCG 94 imply that HCGs require more time to merge than many theories predict, perhaps providing a clue to the puzzle of the abundance of compact groups and the lack of remnants.

This paper benefited greatly from illuminating discussions with J. Bregman, J. Schombert, and A. Evrard. This material is based upon work supported under a National Science Foundation Graduate Fellowship and NASA grants NAGW-2135 and NAG5-1955. 
Table 1. Fitted spatial properties of X-ray emission

\begin{tabular}{cccccc}
\hline \hline PHA & center $(\mathrm{J} 2000)$ & radius & $r_{\text {core }}$ & $\beta$ & count rate \\
\hline $2-4$ & $23^{h} 17^{m} 13^{\mathrm{s}} 1$, & $4.2^{\prime}(0.31 \mathrm{Mpc})$ & $0.63^{\prime} \pm 0.05^{\prime}(46 \mathrm{kpc})$ & $0.56 \pm 0.03$ & $0.038 \mathrm{~s}^{-1}$ \\
& $18^{\circ} 42^{\prime} 32^{\prime \prime}$ & & & & \\
$5-9$ & $23^{h} 17^{m} 13.4$, & $5.0^{\prime}(0.36 \mathrm{Mpc})$ & $0.55^{\prime} \pm 0.05^{\prime}(40 \mathrm{kpc})$ & $0.65 \pm 0.02$ & $0.090 \mathrm{~s}^{-1}$ \\
& $18^{\circ} 42^{\prime} 07^{\prime \prime}$ & & & & \\
\hline
\end{tabular}




\section{REFERENCES}

Barnes, J.E. 1989, Nature, 338, 123

Bode, P.W., Cohn, H.N., \& Lugger, P.M. 1993, ApJ, 416, 17

Burns, J.O., Rhee, G., Owen, F.N., \& Pinkney, J. 1994, ApJ, 423, 94

Cavaliere, A. \& Fusco-Femiano, R. 1976, A\&A, 49, 137

Ebeling, H., Voges, W., \& Böhringer, H. 1994, ApJ, 436, 44 (EVB)

Governato, F., Bhatia, R., \& Chincarini, G. 1991, ApJ, 371, L15

Gregg, M.D. 1989, ApJS, 69, 217

Hickson, P. 1982, ApJ, 255, 382

—. 1993, Astrophys. Lett. \& Comm., 29, 1

Hickson, P., Mendes de Oliveira, C., Huchra, J.P., \& Palumbo, G.G.C. 1992, ApJ, 399, 353

Landolt, A.U. 1992, AJ, 104, 340

Mamon, G.A. 1987, ApJ, 321, 622

Mendes de Oliveira, C. \& Hickson, P. 1994, ApJ, 427, 684

Pildis, R.A., Bregman, J.N., \& Evrard, A.E. 1995a, ApJ, 443, 514 (Paper I)

Pildis, R.A., Bregman, J.N., \& Schombert, J.M. 1995b, in preparation (Paper II)

Ponman, T.J., Allan, D.J., Jones, L.R., Merrifield, M., McHardy, I.M., Lehto, H.J., \& Luppino, G.A. 1994, Nature, 369, 462

Schneider, D.P. \& Gunn, J.E. 1982, ApJ, 263, 14

Schneider, D.P., Gunn, J.E., \& Hoessel, J.G. 1983, ApJ, 264, 337

Schombert, J.M. 1986, ApJS, 60, 603

—. 1988, ApJ, 328, 475 
Fig. 1.- Grayscale image of HCG 94 in the V band. North is to the top and east is to the left, and the image is $3.9^{\prime}$ by $4.4^{\prime}(0.28$ by $0.32 \mathrm{Mpc})$. The bright galaxy at the center of the image is galaxy a, the galaxy to the southwest of a is galaxy b, and the remaining five galaxies in the group (four of which are shown) extend to the northeast of a.

Fig. 2.- Radial profiles of the background-subtracted HRI data for HCG 94. (a) Profile for PHA channels 2-4. The background level was 0.0105 counts $\operatorname{arcsec}^{-2}$. (b) Profile for PHA channels 5-9. The background level was 0.0200 counts $\operatorname{arcsec}^{-2}$.

Fig. 3.- V band grayscale image of HCG 94 with HRI X-ray contours overlaid. The scale is identical to that of Fig. 1. The X-ray data from PHA channels 5-9 are smoothed with a gaussian of $\mathrm{FWHM}=7^{\prime \prime}$, and the contours are averaged over the same scale. The lowest contour is at 0.055 counts per $\left(0.275^{\prime \prime} \times 0.275^{\prime \prime}\right)$ pixel, and the highest is at 0.20 counts per pixel. Models of the two brightest galaxies have been subtracted from the optical image, and the stretch was chosen to maximize the diffuse optical envelope. 


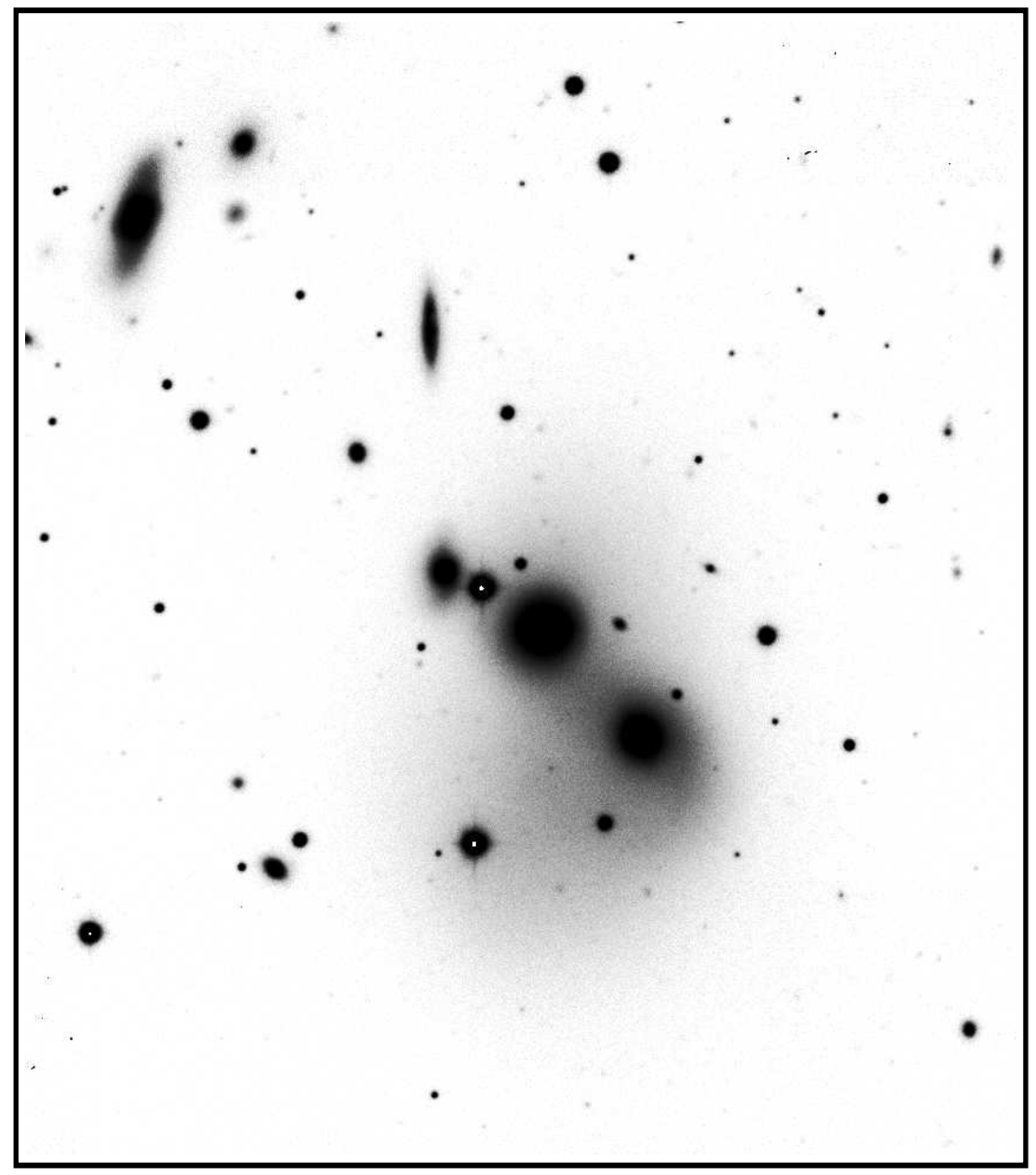




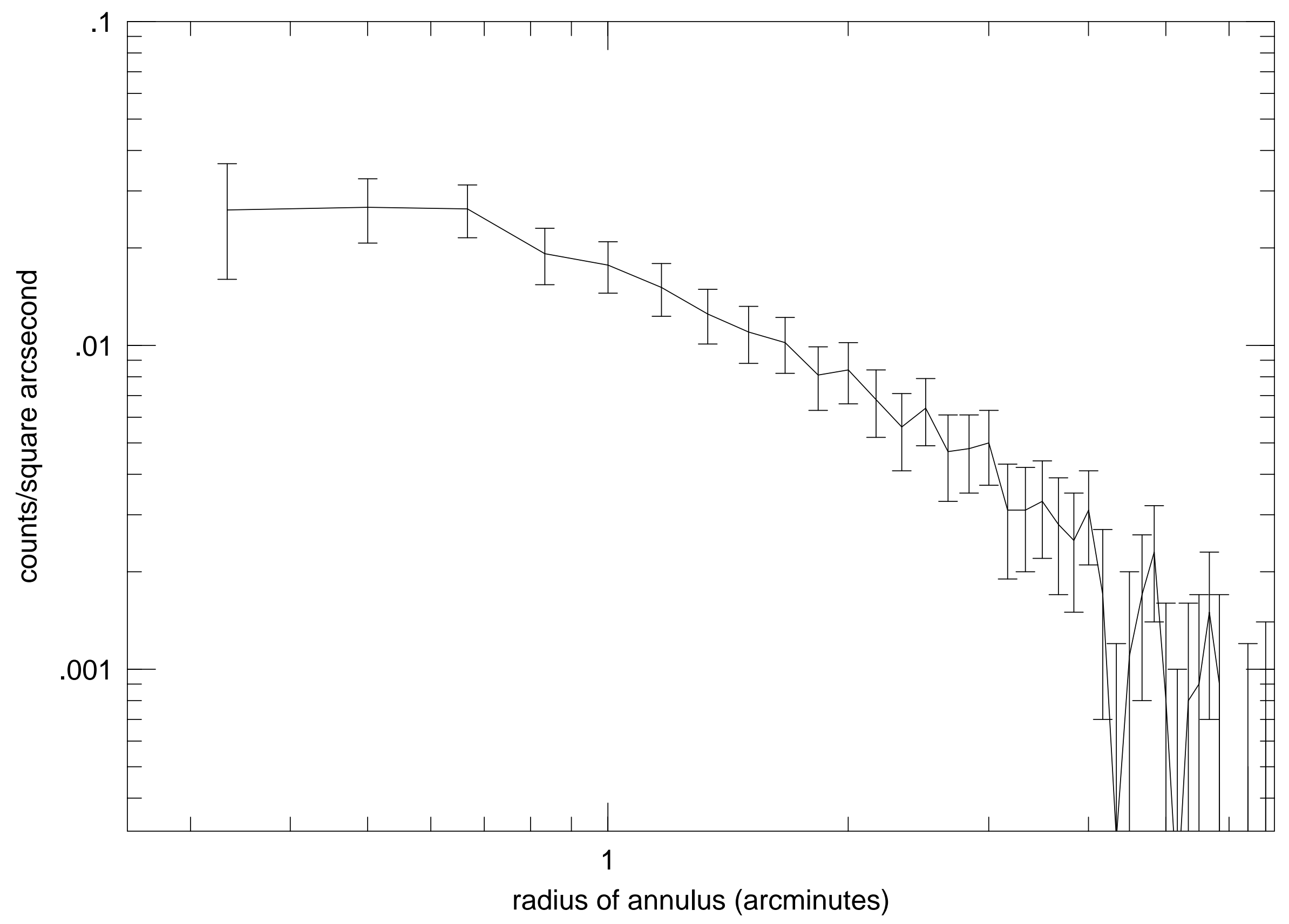




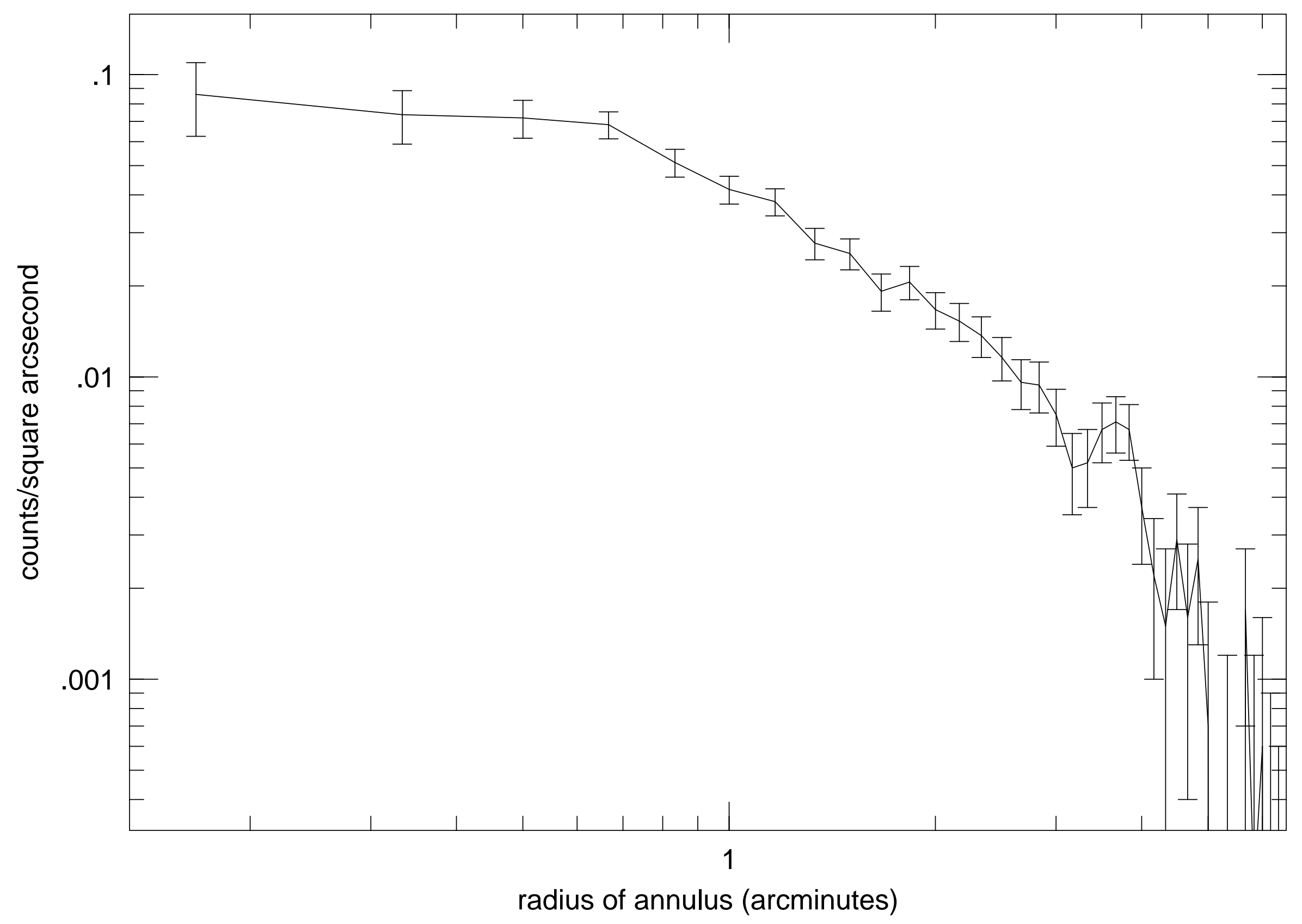




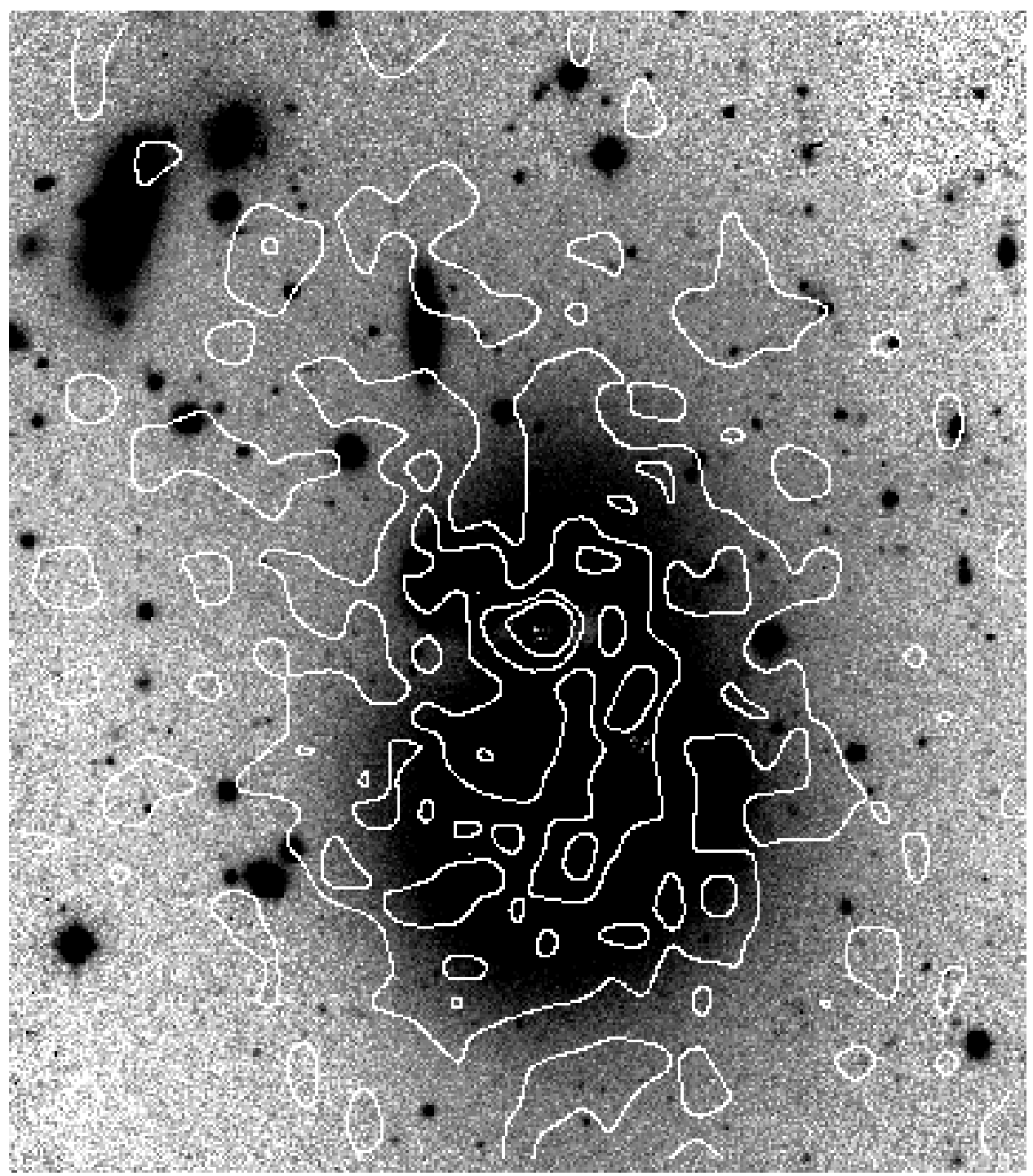

